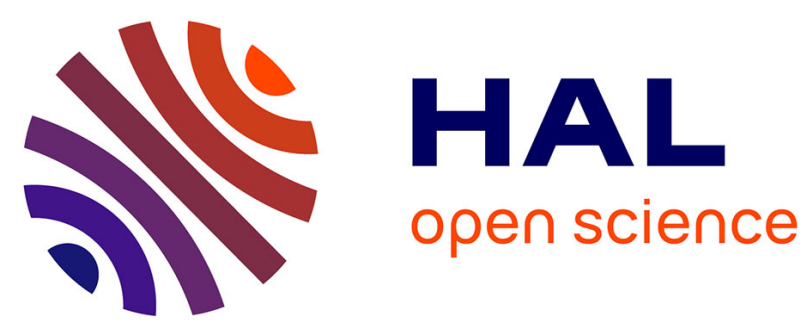

\title{
The Structural and Functional Organization of Ribosomal Compartment in the Cell: A Mystery or a Reality?
}

Elizaveta A Karpova, Reynald Gillet

\section{- To cite this version:}

Elizaveta A Karpova, Reynald Gillet. The Structural and Functional Organization of Ribosomal Compartment in the Cell: A Mystery or a Reality?. Trends in Biochemical Sciences, 2018, 43 (12), pp.938-950. 10.1016/j.tibs.2018.09.017 . hal-01903169

HAL Id: hal-01903169

https://hal-univ-rennes1.archives-ouvertes.fr/hal-01903169

Submitted on 13 Nov 2018

HAL is a multi-disciplinary open access archive for the deposit and dissemination of scientific research documents, whether they are published or not. The documents may come from teaching and research institutions in France or abroad, or from public or private research centers.
L'archive ouverte pluridisciplinaire HAL, est destinée au dépôt et à la diffusion de documents scientifiques de niveau recherche, publiés ou non, émanant des établissements d'enseignement et de recherche français ou étrangers, des laboratoires publics ou privés. 


\section{The Structural and Functional Organization of Ribosomal Compartment in the Cell: a Mystery or a Reality?}

Elizaveta A. Karpova ${ }^{1,2}$ and Reynald Gillet ${ }^{3 *}$

${ }^{1}$ Department of Chemistry, College of Arts and Sciences, University of Alabama at Birmingham, CH266, $90114^{\text {th }}$ Street South, Birmingham, AL, 35294-1240, USA

${ }^{2}$ Department of Physics, Division of Molecular Biophysics and Physics of Polymers, St.

Petersburg State University, Ulyanovskaya 1, St. Petersburg, Russia, 198904

${ }^{3}$ Univ Rennes, CNRS, IGDR (Institut de Génétique et Développement de Rennes) - UMR 6290, F-35000 Rennes, France.

*Correspondence: eak@uab.edu and reynald.gillet@univ-rennes1.fr

Key words: ribosome, polysome, cellular compartment, RNA polymerase, crystals, electron microscopy.

Word count: 4535 


\begin{abstract}
Great progress has been made toward solving the atomic structure of the ribosome, which is the main biosynthetic machine in cells, but we still do not have a full picture of exactly how cellular ribosomes function. Based on the analysis of crystallographic and electron microscopy data, we propose a basic model of the structural organization of ribosomes into a compartment. This compartment is regularly formed by arrays of ribosomal tetramers made up of two dimers that are actually facing in opposite directions. The compartment functions as the main 'factory' for the production of cellular proteins. The model is consistent with the existing biochemical and genetic data. We also consider the functional connections of such a compartment with cellular transcription and ribosomal biogenesis..
\end{abstract}




\section{Introduction}

Most of what we currently know about the fine structural organizations of living cells is the result of conventional electron microscopy (EM) and cryo-electron tomography (CET) study of either macromolecules extracted from cells or whole cells fixed at a certain stage of the life cycle $[1,2,3]$. Three-dimensional structured illumination microscopy of living cells has also yielded interesting data [4]. However, so far no method exists for providing real-time imaging of an intact cell down to its smallest molecules. Nevertheless, certain proven results can form a solid foundation for understanding the structure and functioning of living cells at a quite detailed level. Ribosomes are the main machines for protein production within cells, but a single ribosome cannot do this alone. In fact, cellular protein synthesis needs at least one compartment where ribosomes can work together to translate messenger RNA (mRNA). These structural organizations also protect ribosomes and mRNA from ribonuclease or chemical cleavages. Considering the tremendous amount of ribosomes in actively proliferating cells and the EM data discussed below, we propose a new model for the structural and functional organization of an essential compartment formed by well-organized chains of polysomes. We also consider the functional connections of such a compartment with cellular transcription and ribosome biogenesis.

\section{From ribosomes to polysomes}

Ribosomes were discovered more than a half of a century ago [5]. The main structural organizations of ribosomes have all been found to be similar, even though prokaryotic and eukaryotic ribosomes show substantial differences in size, weight, and molecular composition.

The average size of a prokaryotic ribosome is $200-250 \AA$ and the weight approximately 2.5 $\mathrm{MDa}$, whereas a eukaryotic one is $250-300 \AA$ and weighs from $3.3 \mathrm{MDa}$ in yeast to $4.3 \mathrm{MDa}$ in 
humans [6]. Most of the fine structural organization features of ribosomes were only uncovered in detail in the past 15-20 years (for a recent review, see [7]). Upon interaction with mRNA, ribosomes form higher-order structures named polyribosomes, or polysomes, which efficiently translate genetically encoded information into proteins [8]. Encouragingly, during the last two decades, there have been significant improvements in electron microscopy techniques, particularly in (cryo)-electron tomography [1,9], as well as in complex computer programs for the collection and analysis of EM data $[10,11]$. This progress has yielded new data on the structural organization of polysomes not only in vitro [12-16], but also in living cells $[10,17,18]$.

\section{Basic experimental data support a ribosomal compartment model}

Most cellular functions arise from the interactions between the many different molecular species that inhabit the cells. In sociology, the behavior of a group of people is described not based on the behavior of the individual members of the group, but instead on the interactions of the individuals with each other. In fact, the same basic principle is observed within a cell: individual molecules interact and are spatially arranged within functional modules, making up the 'molecular sociology' of the cell $[19,20]$. Accordingly, this new ribosomal compartment model is based on individual ribosomes working together to form functional ribosomal clusters that thus possess new qualities. Indeed, the intracellular organization into clusters helps explain some of the existing discrepancies between the in vitro and in vivo data, or at least provides an alternative interpretation of these results.

When modeling the ribosomal compartment, at least four major facts had to be considered. First, an extremely large amount of ribosomes are found in actively proliferating cells: in bacteria, 2-3 $\mathrm{x} 10$ per cell [21]. At this concentration, and considering uniform ribosome distribution 
throughout a bacterial cell, the average distance between ribosomes is predicted to be $340-540 \AA$, depending on the phase of bacterial growth [21]. This is in fact about the same as the size of an actual ribosome. Second, bacterial ribosomes purified under gentle conditions [22] are easily assembled into ordered crystal structures (E.A. Karpova, PhD thesis, Tbilisi State University, 1989), [23, 24]. Several EM images of the cross-sections of three-dimensional (3D) crystals of Thermus thermophilus bacteria are given here (Fig. 1a-c) as an example [24]. Milligan and Unwin demonstrated that two-dimensional (2D) crystals are also easily formed during the crystallization of eukaryotic ribosomes in vitro [25]. Third, advances in EM and molecular biology have allowed the reconstruction of electron tomography images of cells that have ordered arrangements of ribosomes during paused translation [18]. On tomograms of polysomeaccumulating bacterial cells, tetramers and long, ordered, double-row chains of densely packed ribosomes are clearly seen (Figs. 1d-e). Notably, on these tomograms the distance between ribosomes is $\sim 25 \mathrm{~nm}$, even smaller than the estimate in the research discussed above [21]. A similar distance between neighboring ribosomes was observed in polysomes from Escherichia coli (E. coli) cell lysate [14]. In fact, at first glance (Fig. 1) it is difficult to say which micrographs were obtained from 3D ribosome crystals, and which were obtained from a cell. We suppose that this similarity is not random, and a certain arrangement of ribosomes close to the ribosomal arrangement in crystals must exist in cells. Finally, when protein synthesis was stopped [26] or paused due to hibernation conditions or hypothermia, 2D ribosome crystals were found in eukaryotes in vivo [27-29].

\section{The structural and functional organization of a cellular ribosomal compartment}

\section{The main unit is a tetramer consisting of two dimers.}


The model presented here (Key Figure ) was designed so that its main unit is a ribosomal tetramer, and a set of ordered tetramers surrounds mRNA to form a polysome. Tetramers were chosen because they are clearly visible on EM images of 2D crystals [25], 3D crystals (Fig. 1ac), and inside the cell [18]. Each tetramer in the model is assembled by two dimers that are flipped over relative to one another. Such a dimer configuration is based on a filtered EM image showing negatively stained ultrathin cross-sections of 3D ribosome crystals of $T$. thermophilus [24,25], shown here at a higher magnification (Fig. 3). This filtered image clearly demonstrates with $70 \AA$-resolution that the ribosomal tetramer is formed by two dimers. One dimer faces in the proper translational direction, while the other one faces in the opposite direction. Layers of eukaryotic ribosomes facing in opposite directions were also observed by Milligan and Unwin under crystallization conditions [25], and this orientation is in agreement with recent EM images of polysomes reconstructed by Baumeister's group [14]. In fact, during purification of bacterial ribosomes, it is known that ribosomal dimers are purified together with 70S monoribosomes $[22,30]$. Indeed, when bacteria undergo stress (e.g starvation conditions) they downregulate their ribosomal activity through dimerization of $70 \mathrm{~S}$ ribosomes by interacting with stationary-phase factors, producing inactive $100 \mathrm{~S}$ complexes that aid in cell survival [31-33]. In E. coli, dimerization is mediated by the hibernation-promoting factor (HPF) and ribosome-modulation factor (RMF). The 30S small subunits of the two 70S ribosomes interact with each other to form a two-fold symmetrical particle [32]. However, the ribosome dimerization mechanism varies depending on the bacterial species, and this makes a big difference in 30S orientation [31]. Notably, ribosomal interaction with hibernation factors does not always result in dimerization, and the regulation and disassembly of $100 \mathrm{~S}$ ribosomes varies greatly among different bacterial phyla, suggesting that other parameters play a role in the process. For instance, GTPase was 
recently shown to be a key regulator of $70 \mathrm{~S}$ and 100S ribosome homeostasis in Staphylococcus aureus, and only GTP can completely dissociate 100S ribosomal dimers [34]. We should emphasize that GTP is the main source of energy for ribosome translation, and particularly for ribosomal translocation on mRNA.

Ribosome dimerization has been observed not only in bacteria [35], but also in mammalian cells [36]. As mentioned above, 2D ribosome crystals formed by ribosomal dimers/tetramers have been found in eukaryotes under stress conditions (hibernation and hypothermia) [27-29]. Ribosomal tetramers are easily isolated along with eukaryotic monoribosomes [25,29]. Importantly, these eukaryotic tetramers are active in the poly(U)-translation system, showing that they can synthesize normal proteins $[25,29]$. The ability of ribosomes to form dimer/tetramer structures shows the high affinity of ribosomes not just to mRNA but also to one another. It is therefore not random that an asymmetric unit of many 3D prokaryotic and eukaryotic ribosome crystals are dimers, and that the crystal cell unit is often formed by ribosomal tetramers $[23,24,37,38]$.

\section{Compartmental links to transcription}

To ensure optimal gene expression, transcription and translation must be synchronized $[39,40]$. However, just because transcription and translation are linked in time and space [41], this does not mean that the two events occur in the same compartment. In fact they occur in different cellular compartments in eukaryotic cells: the nucleus supports transcription, while translation takes place in the cytoplasm. This supports the idea that a separate ribosomal compartment occupies a considerable part of the cell during intensive cell growth.

Similarly, several examples of the compartmentalization of transcription and translation have been observed in prokaryotic cells. For instance, at high growth rates both events occur 
predominantly in separate sub-cellular domains in Bacillus subtilis, although there is no sharp boundary between the two compartments [42]. In the same way, strong nucleoid-ribosome segregation was demonstrated during the entire cell cycle in E. coli $[43,44]$. The location of transcription (nucleoid) and translation (ribosomes) in two compartments does not exclude their cooperation and tight space connection (see Fig. 2), which are modified during different phases of cell growth [44].

In both prokaryotic and eukaryotic cells, a ribosome initiates mRNA translation at the interface between two compartments (translational and transcriptional), where most active RNA polymerases (RNAPs) are localized [42-46]. We propose that the ribosomes that initiate mRNA translation and bind RNAPs are near the compartment's boundary so that they can provide the platform for further assembly of long chains of ordered ribosomes that will participate in translation (Fig. 2). An active complex is formed between RNA polymerase, initiating ribosomes, and freshly synthesized mRNA fragments (see details in Fig. 2a). By interacting with other ribosomes from the cluster, mRNA can form a helix or a zigzag (Fig. 2a and Box 1). Earlier, some EM images of polysomes were interpreted as linear zigzags, and others as linear helices or even as "collapsed rings" $[12,17]$. The final mRNA configuration is dependent on how it interacts with the second, third, and following tetramers. Importantly, mRNA can move through the cluster of tetramers alone or in a binding-tandem with another mRNA. In the latter case, the $3^{\prime}$ terminus of one mRNA interacts with the 5 ' terminus of the other one (see Fig. 2a, 3rd array).

Considering an ordered cluster of ribosomes, we assume that clusters of tetramers ordered within a compartment (Fig. 2a) would not be drastically altered in places where mRNA translation is already complete (see Box 2). Indeed, large changes in the configuration of the compartment 
would be detrimental, especially in terms of energy costs. Moreover, we suppose that exponential cell growth is typically possible if there is a translational compartment that is already organized and ready for the translation of a bulk amount of different proteins in rapidly growing cells.

\section{Compartmental boundaries}

To provide the platform for the further assembly of the long chains of ordered ribosomes involved in translation, the lead ribosomes that initiate mRNA translation and bind RNAPs should connect tightly to the compartment's boundaries (Fig. 2a). We suggest that these boundaries could at least in part be formed by lipids. In eukaryotes, a pool of ribosomes produces secretory and membrane proteins that are either transported across or inserted into the membrane. Membrane-bound ribosomes synthesize these proteins in the eukaryotic endoplasmic reticulum (ER) membrane. Protein synthesis, transport and N-glycosylation are coupled at the mammalian endoplasmic reticulum (ER) by complex formation between the ribosome, the Sec61 protein-conducting channel and the oligosaccharyltransferase [48]. On the other hand, there is no sharp membrane boundary between transcriptional and translational compartments in prokaryotes. Prokaryotic membrane-bound ribosomes are connected to the plasma membranes of eubacteria and archaea $[49,50]$. Co-translational translocation of secreted or membrane proteins by ribosomes occurs through a conserved protein-conducting channel, Sec61 in eukaryotes, and $\mathrm{Sec} \mathrm{Y}$ in prokaryotes. This process is mediated by the signal recognition particle (SRP) and its membrane receptor [50]. It is not known how segregation of nucleoid and ribosome-rich areas occurs, and how the boundaries are formed. However, membraneless organelles and small cellular compartments were recently suggested to be formed by a liquid-liquid phase separation 
[51-54]. We assume that a similar process could take place in prokaryotes when forming translational and transcriptional compartments.

We presume that the highly ordered networks of eukaryotic tetramers (2D ribosomal crystals) observed in the cells of hibernated or hypothermic eukaryotes in early EM experiments [27-29] must be formed by ribosomes attached to the compartment's boundary. Therefore, EM images of 2D eukaryotic ribosome crystals [27-29] should reveal the structural order in a cell before most translation starts and the cell begins to grow again after hibernation. We also presume that lead ribosomes are already present in newborn cells, ready to synthesize ribosomal proteins which will be assembled into new ribosomes. Indeed, we obtained an interesting EM image showing the final step of bacterial cell division. This clearly shows ribosomal tetramers near the membrane separating two newborn cells during cytokinesis (Fig. 1e)

\section{The ribosomal compartment and ribosomal biogenesis}

The arrival and/or replacement of ribosomes within the polysomal compartment must be constant and dynamic. In eukaryotes, ribosome assembly occurs in various cellular compartments. Preribosomal particles undergo a process of maturation which begins in the nucleus after transcription, continues in the nucleoplasm, is followed by nuclear export, and is completed by quality control in the cytoplasm [55]. In growing yeast cells, about 25 pre-ribosomal particles are exported into the cytoplasm each minute [56]. In bacteria, fewer components and factors are needed for this co-transcriptional assembly process. Ribosome biogenesis occurs, we assume, in or close to the nucleoid area where free ribosomal subunits have been observed in live E.coli cells $[43,44]$. The process is highly efficient and rapid, with a single ribosome taking about 2 minutes to be produced [57]. Ribosomal proteins, which are supposed to be synthesized in the 
ribosomal compartment, might rapidly diffuse to the nucleoid area where the ribosomal RNA (rRNA) is transcribed because of the co-transcriptional nature of the assembly process. Several mechanisms pair ribosome biogenesis and protein translation (Fig. 2c), and we observe an autoregulatory feedback of translational control between the two processes. Indeed, when the number of actively translating ribosomes is lowered, there is an increase in both rRNA production and ribosomal protein synthesis, and vice versa [58,59]. This demonstrates the close and direct relationship that exists between ribosomal biogenesis and the preservation of the translational compartment.

\section{Discussion of the model}

The model we present here is in agreement with the results of many in vitro [12-15] and in vivo $[10,17,18]$ polysome studies. In this model, large quantities of ribosomes are tightly packed into a compartment, which resembles a crystal structure having ordered large ribosomal areas. The ribosomal compartment is in some ways reminiscent of an organized electronic circuit [60], in which diodes work together to permit the flow of current in one direction. Similarly, very precisely organized ribosomes allow mRNA to be sent in one direction, and in both of these cases, energy is efficiently and safely transformed into new products. Ribosomal clusters inside cells have been visualized by EM tomography both in prokaryotes $[10,18]$ and eukaryotes $[3,15,17]$. These tomographic studies indicate that ribosomal arrangement is not chaotic in cells: ribosomes adjust their configurations according to their neighbors [17,18].

\section{The impossible closed-loop mRNA configuration}


Our model does not support the widely discussed closed-loop model of cellular protein translation [10,61-65]. The "closed-loop" model suggests that polysomal mRNA is formed by interactions between the two mRNA ends ( $5^{\prime}$ interacting with $3^{\prime}$ ), which would result in a closedlike loop or circular structure, with ribosomes moving along this circular mRNA to synthesize proteins. However, there is no direct structural evidence for this mechanism of translation in a cell [17].

Our model is in agreement with data showing that the $3^{\prime}$ end of mRNA is localized close to an mRNA 5 ' end $[64,65]$, but also showing that, importantly, this may actually be the 5 ' end of another mRNA molecule. In this scenario, mRNA not only form circles, but also creates long mRNA chains. As soon as the leading $5^{\prime}$ terminus of the mRNA strand attaches a ribosome from the ribosome cluster (and interacts with the pool of molecules needed for initiation and elongation), synthesis of a protein begins, with the 5' leading mRNA terminus going on to bind to the second ribosome, third one, and so on. Remarkably, it is not necessary for the mRNAs to be identical, and mRNAs that code different proteins may connect to each other. In this case, two or more proteins can be synthesized by the same set of ribosomes. Such a possibility was recently demonstrated by Morisaki and co-workers, who showed that $5 \%$ of ribosomes simultaneously translated two different proteins [66]. Connection of the 3 ' terminus of one mRNA molecule with the $5^{\prime}$ terminus of another newly synthesized mRNA, as we suggest here in our model, is in agreement with earlier data, which demonstrate that the terminating ribosome usually launches the next round of translation $[12,61,63]$. It is noteworthy that "closed-loop" polysomes were not found in prokaryotic and eukaryotic cells by recent electron tomography experiments $[10,17,18]$. We assume that the formation of circular mRNA is possible in solution (in vitro) when mRNA concentrations are low and the two ends of the same mRNA are in close 
proximity. The suggested "linear" polysomal configuration in our ribosomal compartment model is consistent with recent EM studies of ribosomal arrangements inside of cells in situ $[14,17,18]$. The linear zigzag or helix polysomes (Box 3) can be easily formed from the set of ribosomes shown in Fig. 2. Although the natural tendency of mRNAs in polysomes is to bend, leading to characteristic shapes such as the spirals or hairpins observed in fibroblasts or thyroid epithelial cells [67], this supramolecular organization does not contradict our model within the overall organization of a large ribosomal compartment.

\section{Why is the basic unit of the model a tetramer?}

We propose that the ribosomal tetramer plays a major role in translation, and that two oppositely oriented dimers form this tetramer. Dimers of the tetramer can lie in the same plane (as shown in Fig. 2), one dimer of the tetramer can be slightly offset on the Z-axis (maybe on $1 / 4$ or $1 / 2$ of a packing unit), or the packing of several such dimers can also be considered as a well-organized spiral. Interestingly, the recent in vitro molecular reconstruction of a large eukaryotic polysome shows a very compact helical structure with about four ribosomes per turn [15]. Importantly, in our model the tetramer's two dimers face in opposite directions, with a complete $180^{\circ}$ rotation around the Z-axis (Fig. 2). This configuration was chosen based on a filtered image (Fig. 3) in an attempt to explain why EM images showed differing results for in vitro polysomes. In some, two parallel rows of densely-packed ribosomes face each other top-to-top (parallel), while in others there were two rows of ribosomes that faced in the top-to-bottom (antiparallel) direction [14]. Images of polysomes with anti-parallel rows were previously interpreted as being hairpins or collapsed circles [67]. Our model satisfies both EM results: mRNA is surrounded by two adjoining ribosome rows facing in the same direction, and by two rows in the opposite direction. 
Opposite-direction dimers might result in more flexible protein synthesis. For instance, when mRNA arrive from opposite sides, one dimer is activated, while the other dimer becomes inactive. Data from Nevo-Dinur et al. from fast-growing E. coli cells [68] shows that cytoplasmic and membrane proteins are translated in different regions, with the mRNA coding for membrane proteins at the membrane, and the mRNA coding for cytoplasmic proteins distributed throughout the cytoplasm. In fact, E. coli mRNA can contain information that helps to localize the corresponding proteins in the cell [68]. The translation of membrane and cytoplasmic proteins in the different areas of the ribosomal compartment may therefore be predetermined not only by their close proximity to the membrane, but also by the fine structural organization of tetramers inside of the compartment's subdivisions.

We cannot exclude the idea that perhaps tetramers can only be formed by dimers facing in the same translational direction in certain areas. Images of ribosomes clustered inside cells were recently reconstructed with parallel and anti-parallel rows of ribosomes [18] seen using EM tomography, template matching, and 3D modeling. Densely packed rows of ribosomes were observed inside the cells, perpendicular to planar tetramers (see Fig. 6 in [18]), with four rows of ribosomes connected to the planar tetramer interpreted as two hairpins. However, these images are also in agreement with our model, where two adjoining rows of ribosomes face in opposite directions, while two additional rows of ribosomes face in the same direction directly next to them (Fig 2a). It should also be mentioned that in EM tomograms, some purified polysomes look as if they are partially covered by more than just two rows of ribosomes $[12,69]$.

\section{Channels roaming the ribosomal compartment}


Our model features channels for mRNA and for the flow of small molecules and macromolecular complexes inside an ordered network of ribosomal tetramers (Fig. 2 and 3). These channels were proposed based on EM images of crystal cross-sections (Fig. 1), plus a filtered one (Fig. 3, [24]). Based on experimental data, the mRNA channels (Fig. 2a and Fig. 3, red arrows) are located near the head of a small (30S) ribosomal subunit near the platform [70,71]. Other channels (Fig. 2a and Fig. 3, green arrows) can be used for small molecules (flow of macromolecules and macromolecular complexes) and also, possibly, for macromolecular complexes like chaperones that aid in protein folding. 'Head-to-head' orientation is clearly seen on the filtered image of crystal cross-sections (Fig. 3). Accordingly, our model satisfies the in vitro results of Myasnikov et al. [15], who suggested that the mRNA strand should connect the ribosomes together, forming them into a continuous twisted channel that follows the helical symmetry of the polysome. As we suggested, this channel is already formed by ribosomes interacting with one another due to high affinity.

\section{Translation termination and initiation within the ribosomal compartment}

We should emphasize that the spatial organization of ribosomes described here (Fig. 2a) does not encourage the formation of terminating mRNA hairpins during translation. This is consistent with the ability of ribosomes to block intrinsic termination [39], and in agreement with data that suggests that the average speed of translation is uniform across the length of coding regions for

all genes in the same cell. Translation speed does not depend on the length and amount of encoded proteins [72], and it varies according to cell types and stages [73]. It is noteworthy that when analyzing ribosome profiling data, Michel and Baranov [72] concluded that the density of 
ribosomes on mRNA molecules should be similar for both mRNA covered by fast translating ribosomes and for those containing paused ribosomes, and our model supports this conclusion.

Our model is also consistent with the surprising fact that the involvement of termination factors in the translation process is independent of the entire termination event [62]. The model is in agreement with Ribo-seq analysis showing that multiple translation initiation sites are responsible for the translation of numerous open reading frames (ORF) upstream of the protein coding direction [72].

Remarkably, our model presumes direct interaction between an RNAP and an initiating ribosome in prokaryotes, which means that there is no mRNA transport through the cytoplasm, and synthesized mRNA is protected from degradation. This is in agreement with recent structural ribosome-RNAP studies in the laboratories of Korostelev [74] and Blaha [75], and with the finding that RNAP and the E .coli ribosome can form an "expressome" transcribing-translating complex [76]. The expressome continuously protects the $\sim 30$ nucleotides of mRNA while it moves from the RNAP active center to the ribosome decoding center [76].

In our model, most active RNA polymerases are localized on the boundary between the transcriptional (DNA) and translational (ribosome) compartments. The study of bacterial cells by 3D structured illumination microscopy has revealed dense clusters of transcribing RNAPs almost exclusively at the nucleoid periphery [4]. Earlier, Lewis et al. [42] used fluorescence microscopy on fixed bacterial cells to demonstrate that there is only a slight overlap between the signals representing RNAPs and those from the ribosomal compartment. This was confirmed in living cells by single-molecule fluorescence imaging [43]. Also, an entropy model suggests that transcription initiation may occur preferentially near the ribosome-rich periphery [45]. 


\section{Concluding remarks}

Our hypothetical model for the structural and functional organization of the cellular ribosomal compartment is in a good agreement with the current experimental data for both prokaryotic and eukaryotic cells (see Box 3). We discuss here the formation of the intracellular ribosomal compartment and, in parallel, do a comparison of this compartment with the in vitro growth of ribosomal crystals. We also emphasize that $2 \mathrm{D}$ ribosome crystals were observed in vivo in eukaryotes during hibernation and hypothermia. Crystal growth occurs through the process of phase separation, and the creation of compartments inside of a cell might develop in the same way. It has recently been suggested that liquid-liquid phase separation contributes to the formation of many biomolecular condensates such as eukaryotic RNA granules [51,52]. In the 1960s, Bayer called layers of 2D hypothermic crystallized ribosomes "mysterious cell components" [27]. Today, due to the development of molecular biology and high-resolution structural analysis, the function of these crystalline layers has become more apparent, and there is growing evidence that the ribosomal crystal network is an important intracellular functional stage of the cell. It is extremely difficult to work with living cells, which rapidly react to environmental changes and external stimuli such as temperature shock. Recent advances in cryofocused ion beam (FIB) techniques make now possible to directly observe frozen hydrated cells and tissues by transmission electron microscopy, producing in situ high-resolution 3D snapshots. Baumeister's group applied this technique to HeLa cells in a single in situ data set, and they were able to confirm the close-packed arrangement of membrane-bound ribosomes into polysomes, having a topology dictated by membrane-association [3].

We hope that further development of electron microscopy and other new methods for the observation of fine structures within living cells will provide more evidences for this model. We 
believe that the ribosomal compartment model presented here is critical to the further understanding of the functioning of the cell as a whole system, and it will certainly have an impact on our understanding of "What is the cell?".

\section{Author contributions:}

E.A.K. initiated the work, analyzed the data, and wrote the manuscript. R.G. provided critical feedback throughout, and support in writing the manuscript.

\section{Competing financial interests:}

The authors declare no competing financial or non-financial interests.

\section{Acknowledgments:}

We apologize to those authors whose work has not been cited due to space limitations. E.A.K. would like to dedicate this paper to parents and teachers. E.A.K. is particularly thankful to Dr. Y.S. Tarakhovsky (ITEB, Pushchino, Russia) for past collaboration, encouragement, and support; Dr. K.E. Wilson (Stanford University, CA), A. Tinari for help in preparation of the manuscript; and Elsevier Publishing for allowing use of pictures from previous publications. At UAB, we also thank Dr. J. Zhang for his interest in this work and Dr. N. Schormann for useful discussions in the area of X-ray crystallography. 


\section{REFERENCES}

1. Medalia, O. et al. (2002) Macromolecular architecture in eukaryotic cells visualized by cryoelectron tomography. Science 298, 1209-1213

2. Orlov, I. et al. (2017) The integrative role of cryo-electron microscopy in molecular and cellular structural biology. Biol Cell 109, 81-93

3. Mahamid, J. et al. (2016) Visualizing the molecular sociology at the HeLa cell nuclear periphery. Science 351, 969-971

4. Stracy, M. et al. (2015) Live-cell superresolution microscopy reveals the organization of RNA polymerase in the bacterial nucleoid. Proc Natl Acad Sci U S A 112, E4390-E4399

5. Palade, G. E. (1955) A small particulate component of cytoplasm. J. Biophys. Biochem. Cytol. 1, 59-68

6. Melnikov, A. et al. (2012) One core, two shells: bacterial and eukaryotic ribosomes Nat Struct Mol Biol. 19, 560-567

7. Frank, J. (2017) The mechanism of translation. F1000 Res. 6, 198

8. Warner, J.R. et al. (1962) Electron microscope studies of ribosomal clusters synthesizing hemoglobin. Science 138, 1399-1403

9. Lucic, V. et al. (2005). Structural studies by electron tomography, from cells to molecules. Annu. Rev. Biochem. 74, 833-865

10. Ortiz, J.O. et al. (2006) Mapping $70 \mathrm{~S}$ ribosomes in intact cells by cryoelectron tomography and pattern recognition. J Struct Biol. 156, 334-341

11. Kirmizialtin, S. et al. (2015) Using Molecular Simulation to Model High-Resolution Cryo-EM Reconstructions. Method Enzymol. 558, 497-514

12. Afonina, Z.A. et al. (2013) Topology of mRNA Chain in Isolated Eukaryotic DoubleRow Polyribosomes. Biochemistry (Moscow) 78 (5), 445-454

13. Afonina, Z.A. et al. (2014) Formation of circular polyribosomes on eukaryotic mRNA without cap-structure and poly(A)-tail, a cryo-electron tomography study. Nucleic Acids Res. 42, 9461-9469

14. Brandt, F. et al. (2009) The native 3D organization of bacterial polysomes. Cell, 136, $261-271$ 
15. Myasnikov, A.G et al. (2014) The molecular structure of the left-handed supramolecular helix of eukaryotic polysomes. Nat Commun 5, 5294-5300

16. Kopeina, G.S. et al. (2008) Step-wise formation of eukaryotic double-row polyribosomes and circular translation of polysomal mRNA. Nucleic Acids Res 36, 2476-88

17. Brandt, F. et al. (2010) The three dimensional organization of polysomes in intact cells. Mol Cell 39, 560-569

18. Cougot, N. et al. (2014) Visualizing compaction of polysomes in bacteria. J Mol Biol $426,377-388$

19. Beck, M. and Baumeister, W. (2016) Cryo-Electron Tomography: Can it Reveal the Molecular Sociology of Cell in Atomic Detail? Trends Cell Biol 26, 825-837

20. Robinson, C.V. et al. (2007) The molecular sociology of the cell. Nature 450, 973-982

21. Gouy, M. and Grantham, R. (1980) Polypeptide elongation and tRNA cycling in Escherichia coli, a dynamic approach. FEBS Lett. 115, 151-155

22. Karpova, E.A. and I.N. Serdyuk. (1985) Dissociation of Thermus thermophilus 70 S ribosomes upon a change of magnesium ion concentration, ionic strength and $\mathrm{pH}$ of solution. Mol. Biology (USSR) 19, 1585-1590

23. Karpova, E.A et al. (1986) Crystallization of ribosomes from Thermus thermophilus. Dokl. Acad. Nauk (Moscow) 289, 1263-1266 (English translation, Doklady Biophysics 4, $272-$ 274

24. Karpova, E.A. and Tarakhovsky, Y.S. (2001) Altering the morphology of crystals of a macromolecule without changing the unit cell (A case study of ribosome crystals). J. of Crystal Growth 232, 610-617

25. Milligan, R.A. and Unwin, P.N. (1982) In vitro crystallization of ribosomes from chick embryos. J Cell Biol. 95 (2Pt 1), 648-653

26. O'Brien, L. et al. (1980) Proc Natl Acad Sci USA 77, 2260-2264

27. Byers, B. (1966) Ribosome crystallization induced in chick embryo tissues by hypothermia. J. Cell Biol., 30, C1- C6

28. Unwin, P.N. and Taddei, C. (1977) Packing of ribosomes in crystals from the lizard Lacerta sicula. J Mol Biol. 114, 491-506

29. Byers, B. (1971) Chick Embryo Ribosome Crystals, Analysis of Bonding and Functional Activity In Vitro. Proc. Nat. Acad.Sci.USA, 68, 440-444 
30. Lake, J.A. (1982) Packing of 70S ribosomes in dimers formed at low ionic strength. $J$. Mol. Biol 160, 369-373

31. Franken, L.E. (2017) A general mechanism of ribosome dimerization revealed by single-particle cryo-electron microscopy. Nat Commun. 8, 722-731

32. Polikanov, Y.S. et al. (2012) How hibernation factors RMF, HPF, and YfiA turn off protein synthesis. Science. 336, 915-918

33. Matzov, D. et al. (2017) Nat Commun. The cryo-EM structure of hibernating $100 \mathrm{~S}$ ribosome dimer from pathogenic Staphylococcus aureus. Nat Commun 8, 723-729

34. Basu, A. and Yap, M-N. (2017) Disassembly of the Staphylococcus aureus hibernating 100S ribosome by an evolutionarily conserved GTPase Proc Natl Acad Sci U S A. 114, E8165E8173

35. Yoshida, H. and Wada, A. (2014) The 100S ribosome: ribosomal hibernation induced by stress. Wiley Interdiscip. Rev. RNA 5, 723-732

36. Krokowski, D. et al. (2011) Characterization of hibernating ribosomes in mammalian cells. Cell Cycle 10, 2691-2702

37. Demeshkina, N. L. et al. (2012) A new understanding of the decoding principal of the ribosome. Nature 484, 256-259

38. Ben-Shem, A. et al. (2010) Crystal structure of eukaryotic ribosome. Science 330, 1203-1209

39. Elgamal, S. et al. (2016) Maintenance of Transcription-Translation Coupling by Elongation Factor P. mBio 7, e01373-16

40. Proshkin, S. et al. (2010) Cooperation between translating ribosomes and RNA polymerase in transcription elongation. Science 328, 504-508

41. McGary, K. and Nudler, E. (2013) RNA polymerase and the ribosome, the close relationship. Curr Opin Microbiol 16, 112-117

42. Lewis, P.J. et al. (2000) Compartmentalization of Transcription and translation in Bacillus subtilis. EMBO J. 19, 710-718

43. Bakshi, S. et al. (2012) Superresolution imaging of ribosomes and RNA polymerase in live Escherichia coli cells. Mol Microbiol 85, 21-38

44. Bakshi, S. et al. (2015) Spatial biology of transcription and translation in rapidly growing Escherichia coli. Front Microbiol 6: 636 
45. Mondal J. et al. ( 2011) Entropy-based mechanism of ribosome-nucleoid segregation in E. coli cells. Biophys $J$ 100, 2605-2613

46. Travers, A. and Muskhelishvili, G. (2007) A common topology for bacterial and eukaryotic transcription initiation? EMBO Rep. 8, 147-151

47. Woldringh, C.L. (2002) The role of co-transcriptional translation and protein translocation (transertion) in bacterial chromosome segregation. Mol. Microbiol 45, 17-29

48. Braunger, K. et al. (2018) Structural basis for coupling protein transport and Nglycosylation at the mammalian endoplasmic reticulum. Science 360, 215-219

49. Zimmermann, R. et al. (2011) Protein translocation across the ER membrane. Biochim Biophys Acta, Biomembranes 1808, 912-924

50. Rapoport, T.A. et al. (2017) Structural and mechanistic insights into protein translocation. Annu Rev Cell Dev Biol. 33, 369-390

51. Lin, Y. et al. (2017) Intrinsically disordered sequences enable modulation of protein phase separation through distributed tyrosine motifs. J Biol Chem. 292, 19110-19120

52. Wheeler, J.R. et al. (2016) Distinct stages in stress granule assembly and disassembly. Elife e18413.

53. Babani, S.F. et al.( 2017) Biomolecular condensates: organizers of cellular biochemistry. Nat Rev Mol Cell Biol. 18, 285-298

54. Woodruff, et al. (2017) Organization and Function of Non-dynamic Biomolecular Condensates. Trends Biochem Sci. 43, 81-94

55. Pena, C. et al. (2017) Eukaryotic ribosome assembly, transport and quality control. Nat Struct Mol Biol. 24, 689-699

56. Warner, J.R. (1999) The economics of ribosome biosynthesis in yeast. Trends Biochem. Sci. 24, 437-440

57. Davis, J.H. and Williamson, J.R. (2017) Structure and dynamics of bacterial ribosome biogenesis. Philos. Trans. R. Soc. B Biol. Sci. 372, 20160181

58. Kaczanowska, M. and Ryden-Aulin, M. (2007) Ribosome biogenesis and the translation process in Escherichia coli. Microbiol. Mol Biol Rev., 71, 447-494

59. Zengel, J.M. and Lindahl, L. (1994) Diverse mechanisms for regulating ribosomal protein synthesis in Escherichia coli. Prog Nucleic Acid Res Mol Biol. 47, 331-370 
60. Karpova, E.A. (2005) Could we consider a cell as a Device with a set of right organized crystalline ribosomes? J Biomol Struct Dyn 22 (6), 870

61. Philipps, G. R. (1965) Haemoglobin synthesis and polysomes in intact reticulocytes. Nature 205, 53-56

62. Amrani, N. et al. (2008) Translation factors promote the formation of two states of the closed-loop mRNP. Nature 453, 1276-1280

63. Baglioni, C. et al. (1969) The Role of Ribosomal Subunits in Mammalian Cells. Cold Spring Harbor Symp. Quant. Biol. 34, 555-565

64. Wells, S.E. et al. (1998) Circularization of mRNA by eukaryotic translation initiation factors. Mol. Cell, 2 (1), 135-140

65. Madin, K., T. et al. (2004) Formation of circular polyribosomes in wheat germ cell-free protein synthesis system. FEBS Lett 562, 155-159

66. Morisaki, T. et al. (2016) Real-time quantification of single RNA translation dynamics in living cells. Science 352, 1425-1429

67. Christensen, A. K. and Bourne, C.M. (1999) Shape of large bound polysomes in cultured fibroblasts and thyroid epithelial cells. Anat. Record 255, 116-129

68. Nevo-Dinur, K. et al. (2011) Translation-independent localization of mRNA in E. coli. Science 331, 1081-1084

69. Viero, G. et al. (2015) Three distinct ribosome assemblies modulated by translation are the building blocks of polysomes. J Cell Biol 208, 581-96

70. Evstafieva, A.G. at al. (1983) Localization of $5^{\prime}$ and $3^{\prime}$ ends of the ribosome-bound segment of template polynucleotides by immune electron microscopy. EMBO J 2, 799-804

71. Yusupova, G.Z. et al. (2001) The path of messenger RNA through the ribosome. Cell $106,233-41$

72. Michel, A.M. and Baranov, P.V. (2013) Ribosome profiling, a Hi-Def monitor for protein synthesis at the genome-wide scale. In, Wiley Interdiscip Rev RNA, 4, 473-490

73. Pichon, X. et al. (2016) Visualization of Single Endogenous Polysomes Reveals the Dynamics of Translation in Live Human Cells. J. Cell Biol 214, 649-652

74. Demo, G. et al. (2017) Structure of RNA polymerase bound to ribosomal 30S subunit. Elife. 6 
75. Fan, H. et al. (2017) Transcription-translation coupling: direct interactions of RNA polymerase with ribosomes and ribosomal subunits. Nucleic Acids Res 45, 11043-11055

76. Kohler, R. et al. (2017)Architecture of a transcribing-translating expressome. Science 356, 194-197

77. Andreeva, I. et al. (2018) Translation initiation in bacterial polysomes through ribosome loading on a standby site on a highly translated mRNA. Proc Natl Acad Sci U S A. 115, $4411-4416$ 
Box 1. How does mRNA interact with ribosomes during translation? In our model, two ribosomes in each tetramer face in the correct translational direction, and two face the opposite way. The ribosomes interacting with mRNAs can form a helix or zigzag inside the stacked ribosomal tetramers. The mRNA-ribosome interactions result in the overall polysome configuration.

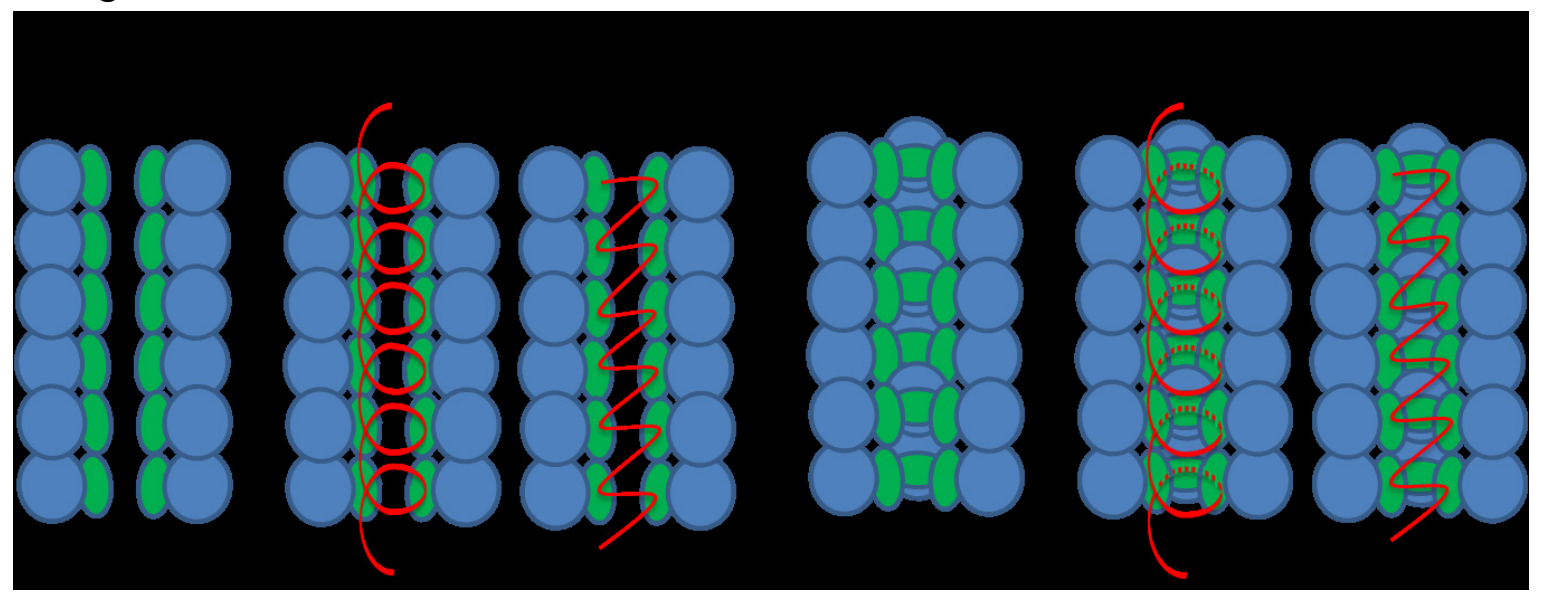

2D and 3D models of the interaction of mRNA with ribosomes. Small ribosomal subunits are green, large ones are blue, and mRNA are red.

\section{Box 2. Thoughts on the stability of ribosomal compartments in living cells.}

When mRNA translation is complete, the ribosomal compartment may change, but it should not collapse. Indeed, that would be detrimental for cells whose ribosomes should be ready for the next round of translation once growth is activated. However, when the cell is degrading, the ribosomal compartment will be also be diminished and eventually destroyed. For an actively growing cell, when the cell has enough nutrients and is at optimal temperatures, it would be energetically detrimental to disrupt this order. We imagine that during the exponential growth phase, when bacterial cells are rapidly proliferating, the translational compartments of the mother cell with "ready to go" ribosomes should be almost equally divided between two daughter cells. This promotes rapid exponential cell growth, since the cells then do not need to recreate translational clusters from scratch. This is consistent with the data of Lewis et al. [42] and Bakshi et al. [43,44] who both examined rapidly growing bacterial cells and detected three major clusters of ribosomes localized outside of the cell nucleoid, predominantly at the cell poles and the mid-cell area of the future division site. However, during the stationary growth phase, the fluorescent signals from GFP-fused ribosomes are weaker and more uniformly distributed, which reflects changes in the arrangement of the ribosomes into cellular clusters [42]. 


\section{Box 3: Evidence supporting the ribosomal compartment model}

1. Ribosomal tetramers and long rows of polysomal clusters are clearly visible on EM images in both prokaryotic and eukaryotic cells $[10,17,18]$.

2. EM data demonstrate that polysomes are arranged as compact staggered or pseudohelical structures in vitro $[14,15]$.

3. 2D-ribosome crystals were found in hibernated eukaryotes [25,27-29], and these ribosomes can be reactivated.

4. Huge numbers of ribosomes are synthesized in fast-proliferating cells [21, 42-44].

5. Initiating ribosomes interact with RNA polymerase, making a ternary RNAP-ribosomemRNA complex [76].

6. Several copies of a protein are translated from a single mRNA (multi-round translation)

7. Terminating a ribosome re-initiates the next round of translation [16].

8. The rates of translation and transcription are nearly the same, since every 3 base pairs are translated into one amino acid [40]. For example, the transcriptional rate is 42 nucleotides/sec and the translational rate is 14 amino acids/sec in the same cells.

9. Most translation occurs on complete mRNAs, which are disconnected from RNAP [43, 44].

10. Ribosomes can easily dimerize when cells encounter stress.

11. Translation and transcription are synchronized [39-41].

12. Our model supports "transertion" of membrane proteins well. In this process, membrane protein synthesis is considered as a non-stop chain of events: transcription, translation, and then translocation [47].

13. The involvement of termination factors in the translation process is independent of the whole termination event [62].

14. Our model has multiple translation initiation sites, in agreement with Ribo-seq analysis [72].

15. The ribosomal compartment protects ribosomes and mRNA from degradation.

16. Our model allows mRNA, translation factors, and newly synthesized proteins to make their way along the pathways through the compartment.

17. RNAP interacts with $30 \mathrm{~S}$ and $50 \mathrm{~S}$ ribosomal particles and with the whole $70 \mathrm{~S}$ ribosome $[74,75]$.

18. A second ribosome, which is next to the one initiating translation is loaded onto the 5 , mRNA untranslated region, before the initiating ribosome moves away from the start codon [77]. 


\section{Figure legends}

Figure 1. Electron microscopy images of ribosomes. (a, b, c) Electron micrographs of positively stained ultrathin ( 40-100 nm) sections of Thermus thermophilus 70S ribosome crystals. Crystals were fixed with $1 \%$ glutaraldehyde, and sections were stained with a saturated solution of uranyl acetate and lead citrate. Ribosomes are dark, and ribosome tetramers and dimers are clearly seen. Scale bar, $200 \mathrm{~nm}$. (d, e) Reconstructed electron tomogram of a $100 \mathrm{~nm}$-thick section of Escherichia coli. Polysomes form double row units or assemblies of tetramers. Scale bar, $100 \mathrm{~nm}$. Pictures (a, b, c) adapted from [24], picture (d) from [18], reproduced here with kind permission from Elsevier, and picture (e) from R. Gillet personal data collection (same experiment as in d).

Figure 2. The intracellular ribosomal compartment model in bacteria. Ribosome tetramers surround messenger RNA (mRNA) and form an ordered 3D structure. Ribosome assembly (biogenesis) occurs in close proximity to the ribosomal compartment. The mature ribosomes are made up of mature ribosomal RNA, specific ribosomal proteins, and assembly factors, connecting translation with ribosomal biogenesis. The ribosomes initiate mRNA translation at the interface between the translational and transcriptional compartments. The RNA polymerase (RNAP) "sits" on the DNA loop and synthesizes mRNA coming from coding genes. As soon as the mRNA reaches a certain length, it interacts with the small ribosomal subunit of an initiating ribosome (ribosome i). The mRNA then binds to the next ribosome (ribosome $\mathrm{i}+3$ ), which is oriented in the proper translational direction. The mRNA $5^{\prime}$ terminus moves further along to the next tetramer, repeating the binding process with the next and then the next ribosome. (a, b) Model views in the Y0Z plane (a, side view) and XZY plane (b, top view). Key: Z1, image in that section which crosses the first double row of ribosomes on level $Z_{1} ; Z_{2}$, the image that crosses the second double row on level $\mathrm{Z}_{2} ; \mathrm{Z} 3$, the image in section $\mathrm{XZ}_{3} \mathrm{Y}$ which crosses the third double row on level $Z_{3}$. (c) Mature ribosomes are obtained from subunit precursors (Pre-50S and Pre-30S). They join the translational ribosomal compartment as monoribosomes or tetramers. Small ribosomal subunits are green, large ones are blue. RNAP is yellow, mRNA helices are red, and the $3^{\prime}$ and $5^{\prime}$ termini of 
mRNA are indicated. Bright green and blue ribosomal tetramers face in the correct translational direction, while the other two light blue ribosomes face the other way. Red arrows indicate the channels for mRNA (and perhaps for other molecules), while green arrows show the channels for macromolecules and big macromolecular complexes, including chaperones.

Figure 3. Optical filtered image of the electron micrograph section of a ribosome crystal. A thin section of ribosome crystals of Thermus thermophilus was stained with uranyl acetate and lead citrate as described in Fig. 1 (a-c). The filtered image was previously published, but is shown here at a higher magnification $(49 \mathrm{~nm})$. The crystal's unit cell in projection X0Y is indicated. Red arrows show the possible channels for mRNAs, which presumably cross the packed ribosomes in direction $\mathrm{Z}$ inside the cell (perpendicular to the X0Y plane). Green arrows show the channels for the flow of molecules and macromolecular complexes within the cell. 


\section{Figure 1}

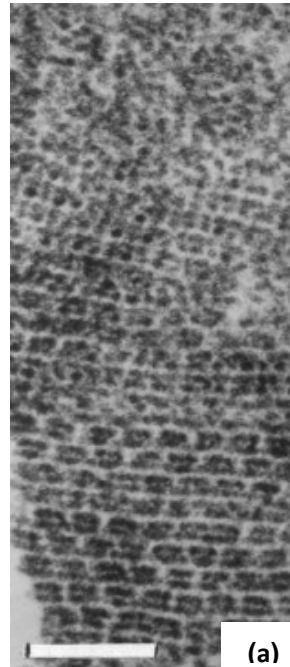

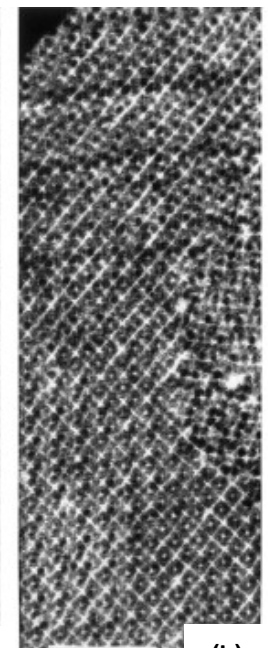

(b)

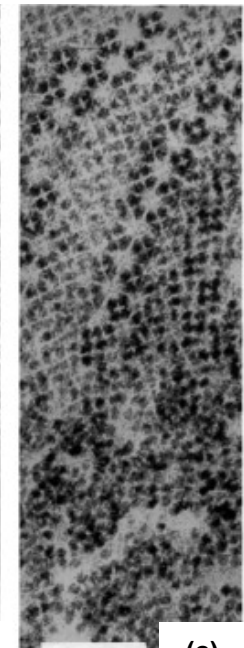

(c)

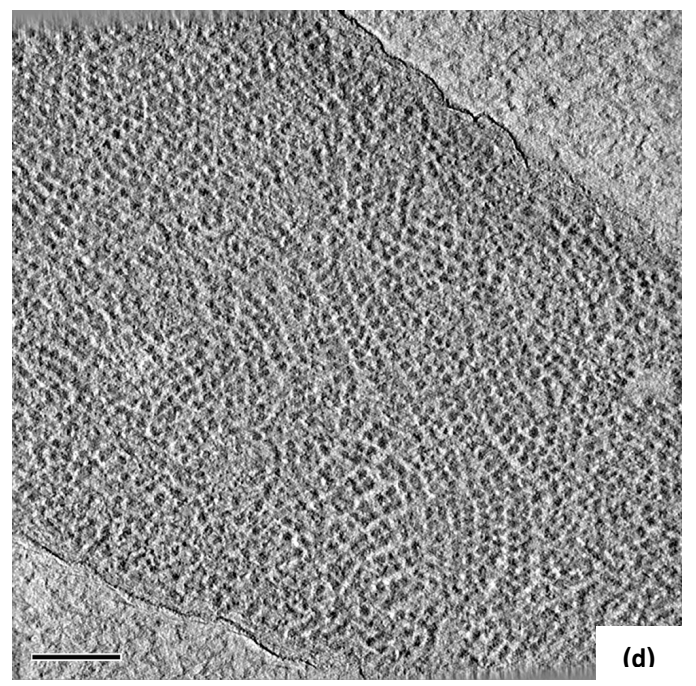

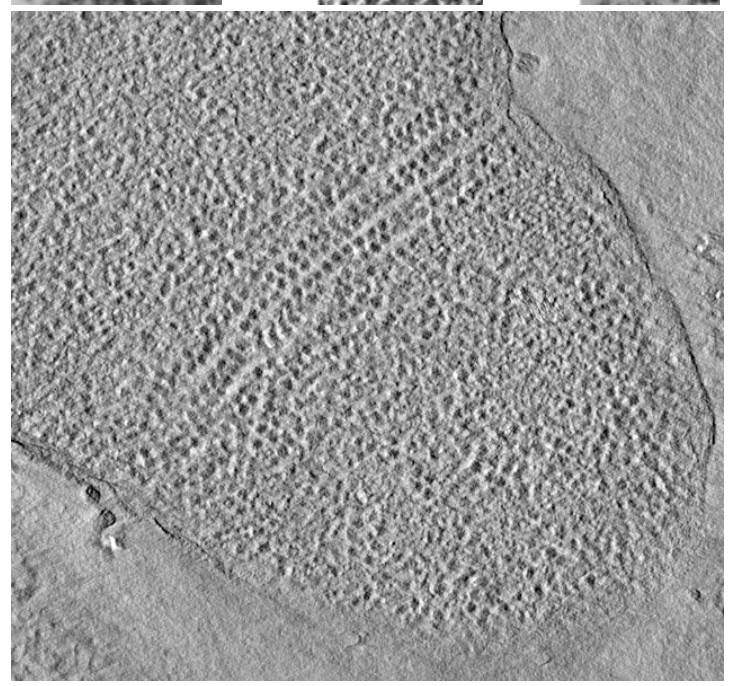


Figure 2

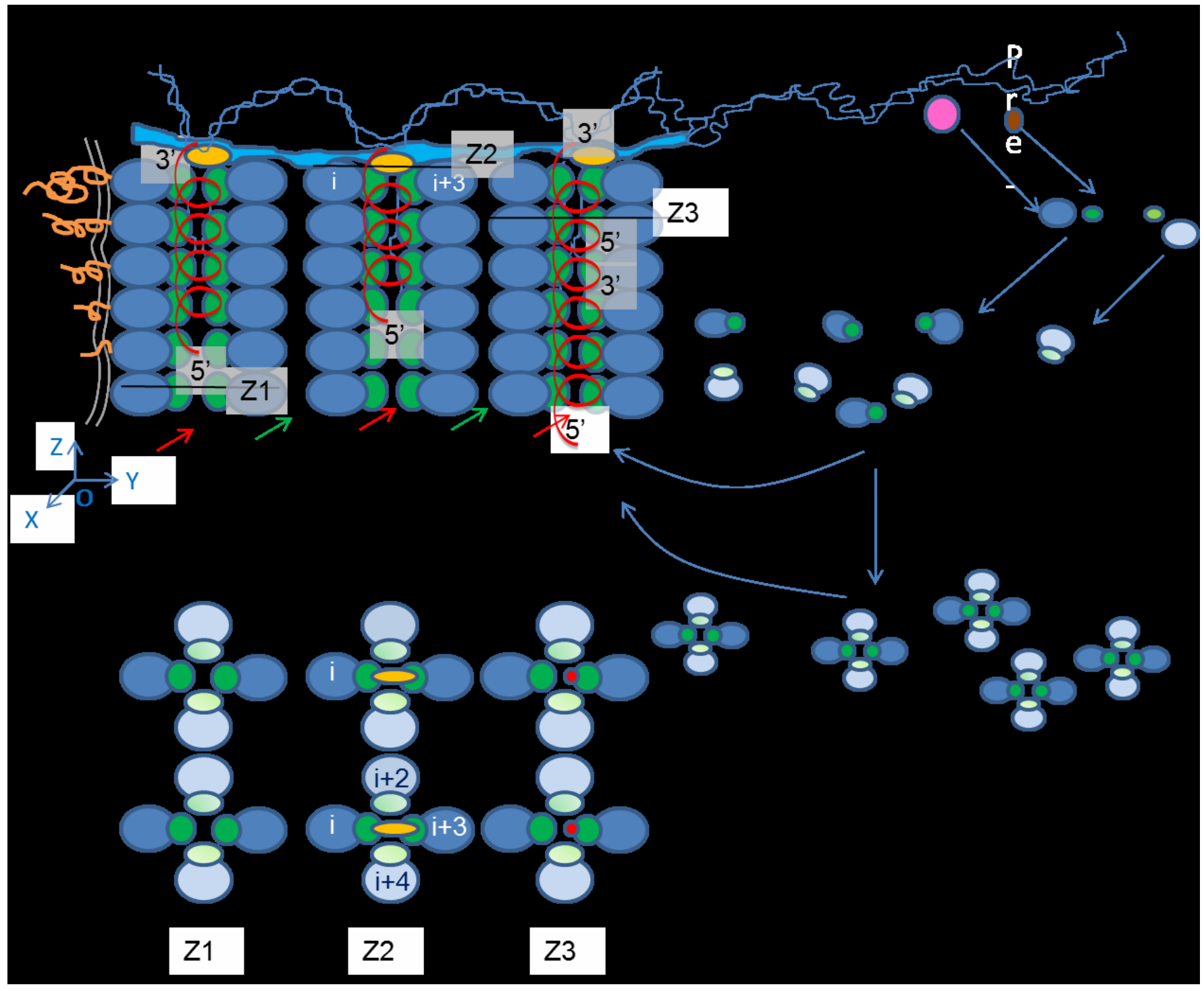


Figure 3

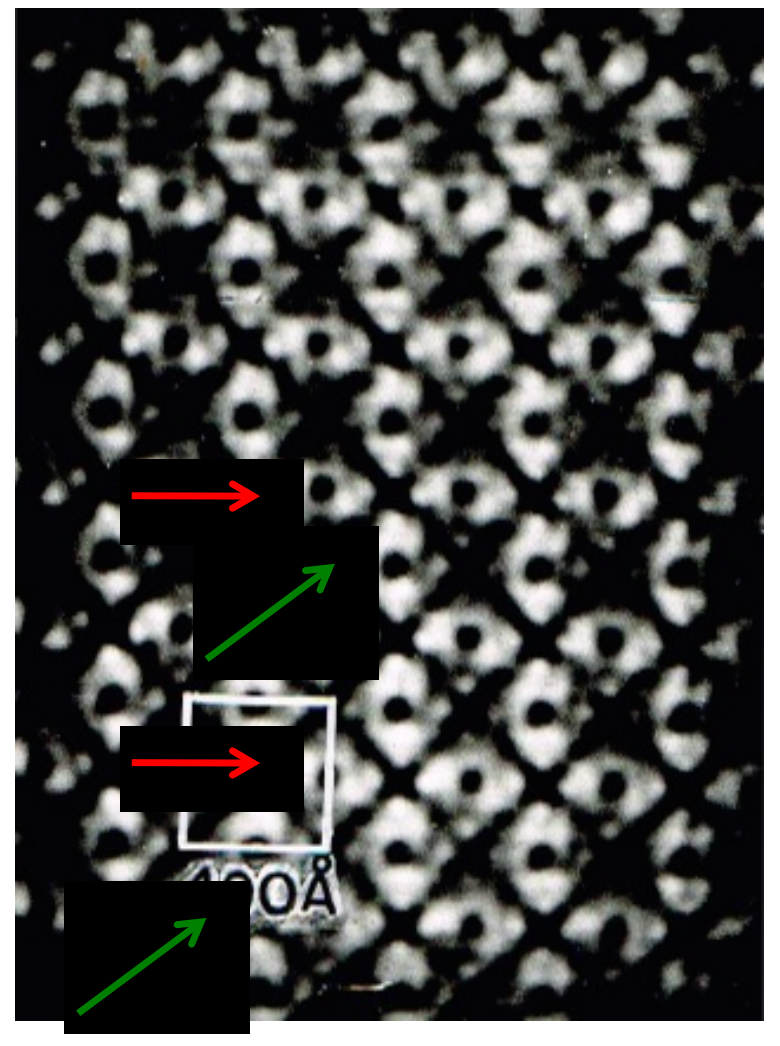

